\title{
The conspicuous absence of X-ray emission from carbon-enriched Wolf-Rayet stars
}

\author{
L. M. Oskinova ${ }^{1}$, R. Ignace ${ }^{2}$, W.-R. Hamann ${ }^{1}$, A. M. T. Pollock ${ }^{3}$, and J. C. Brown ${ }^{4}$ \\ 1 Astrophysik, Univerität Potsdam, Am Neuen Palais 10, 14469 Potsdam, Germany \\ 2 Department of Astronomy, University of Wisconsin, Madison, WI 53706, USA \\ 3 ESA/Vilspa, Apartado 50727, 28080 Madrid, Spain \\ 4 Department of Physics and Astronomy, University of Glasgow, Glasgow G12 8QQ, UK
}

Received 9 December 2003 / Accepted 21 February 2003

\begin{abstract}
The carbon-rich WC5 star WR 114 was not detected during a $15.9 \mathrm{ksec}$ XMM-Newton observation, implying an upper limit to the X-ray luminosity of $L_{\mathrm{X}} \lesssim 2.5 \times 10^{30} \mathrm{erg} \mathrm{s}^{-1}$ and to the X-ray to bolometric luminosity ratio of $L_{\mathrm{X}} / L_{\mathrm{bol}} \lesssim$ $4 \times 10^{-9}$. This confirms indications from earlier less sensitive measurements that there has been no convincing X-ray detection of any single WC star. This lack of detections is reinforced by XMM-Newton and CHANDRA observations of WC stars. Thus the conclusion has to be drawn that the stars with radiatively-driven stellar winds of this particular class are insignificant X-ray sources. We attribute this to photoelectronic absorption by the stellar wind. The high opacity of the metal-rich and dense winds from WC stars puts the radius of optical depth unity at hundreds or thousands of stellar radii for much of the X-ray band. We believe that the essential absence of hot plasma so far out in the wind exacerbated by the large distances and correspondingly high ISM column densities makes the WC stars too faint to be detectable with current technology. The result also applies to many WC stars in binary systems, of which only about $20 \%$ are identified X-ray sources, presumably due to colliding winds.
\end{abstract}

Key words. stars: individual: WR 114 (HD 196010) - stars: winds, outflows - stars: early-type - X-rays: stars

\section{Introduction}

The few major classes of star whose spectral properties are dominated by their mass loss in the form of stellar winds include the hot OB stars, with roughly solar chemical composition and mass-loss rates $\dot{M}_{\mathrm{OB}} \approx 10^{-7} M_{\odot} \mathrm{yr}^{-1}$ and the WolfRayet (WR) stars with $\dot{M} \approx 10^{-5} M_{\odot} \mathrm{yr}^{-1}$. The WR stars come in two main types: WN stars with enriched helium content and deficient hydrogen and WC stars with no hydrogen but strongly enhanced carbon and oxygen. It is thought that $\mathrm{O}$ stars with sufficient initial masses evolve to WR stars, passing through the WN star stage and become WC stars whose own likely end is in gravitational collapse (Woosley et al. 2002).

The mass loss from OB stars is reasonably well explained in the framework of the CAK theory (Castor et al. 1975) of linedriven stellar winds. There has also been progress in the understanding of winds from WR stars. Recent advances in atmospheric modeling demonstrate that the mass loss of WC stars, like that of $\mathrm{OB}$ and $\mathrm{WN}$ stars, is also generally explicable by radiative driving (e.g. Gräfener et al. 2002).

However, the X-ray emission of OB and WR stars remains enigmatic. Discovered with the EINsTeIn observatory (Seward et al. 1979; Harnden et al. 1979), soft X-ray emission from hot

Send offprint requests to: L. M. Oskinova,

e-mail: 1ida@astro.physik.uni-potsdam.de stars was initially attributed to shocks due to the intrinsic instability of the radiative driving mechanism (Lucy \& White 1980; Lucy 1982). Further developments of the theory by Feldmeier et al. $(1997 \mathrm{a}, \mathrm{b})$ were reasonably able to reproduce the lowresolution spectra of O stars observed by the Rosat observatory but are now facing new challenges with the recent advent of high-resolution spectroscopic data from the XMM-Newton and Chandra grating spectrometers.

Application of the theory to X-ray production in the highdensity winds of WR stars has yet to be done in detail. Importantly, however, Gayley \& Owocki (1995) have considered the effects of multiple scattering in WR winds for the wind instability mechanism. They found that multiple scattering has a suppressing effect on the intrinsic instability that is normally thought to explain the X-ray production of the less dense winds of OB stars. However, a significant residual instability remains and is sufficient to produce extensive structure in the wind. Theoretically, this process may lead to shock formation and plasma heated to X-ray emitting temperatures of few million Kelvin. Arguably, the presence of lines of highly ionized species such as OvI in the UV spectra of WR stars indicates the presence of $\mathrm{X}$-ray photons in the line-forming regions of the wind (e.g. Gräfener et al. 2002).

The study of X-rays from single WR stars and their spectral properties provides much needed new insight into 


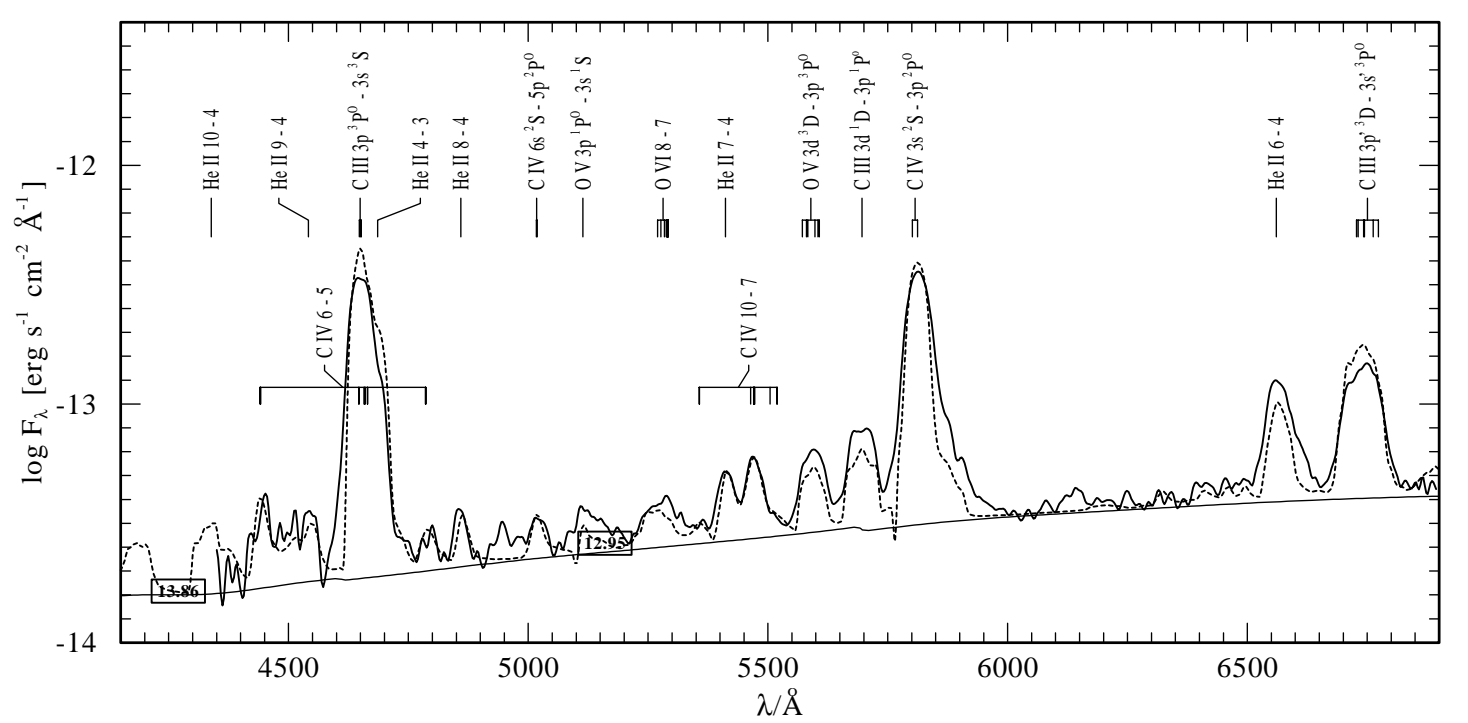

Fig. 1. Observed visual spectrum (solid line) of WR 114 from the atlas of Torres \& Massey (1987), compared to a synthetic model (dotted line) calculated with the Potsdam non-LTE stellar wind code (Gräfener et al. 2002). The thin smooth line is the model continuum. The model parameters are: effective temperature $T_{*}=80 \mathrm{kK}$, luminosity $\log L_{*} / L_{\odot}=5.2$, mass loss rate $\log \dot{M}=-5.0 M_{\odot} \mathrm{yr}^{-1}$, terminal wind velocity $v_{\infty}=2000 \mathrm{~km} \mathrm{~s}^{-1}$, chemical composition He:C:O =55:40:05, and a clumping contrast of $D=10$. The calibration of the observation is confirmed by $v, b$ photometry (small boxes). The synthetic spectrum is scaled with the distance modulus of WR 114 (11.5 mag from the membership in the Sgr OB1 association) and reddened with $E_{b-v}=1.2 \mathrm{mag}$ (corresponding to $E_{B-V}=1.45 \mathrm{mag}$ ) in order to reproduce the observed slope.

the understanding of stellar winds. WR stars have short lifetimes and, although evolutionarily linked to $\mathrm{O}$ stars and thus located in the same OB stellar associations, are relatively rare and distant. Most WR stars are X-ray faint and thus hard to detect. Among the exceptions are the binary systems, where stronger and harder X-ray emission due to wind-wind collisions suggests criteria for distinguishing single and double stars. Unfortunately, little is learned about the X-rays in the WR wind itself from such systems.

In this paper we concentrate on the study of single WC stars. On average, the WC stars, with absolute visual magnitudes not less then $M_{\mathrm{v}} \approx-4.5 \mathrm{mag}$ (vander Hucht 2001) are less luminous than the $\mathrm{O}$ and $\mathrm{WN}$ stars from which they evolve because of the reduction in mass during evolution and the very high effective temperatures of up to $10^{5} \mathrm{~K}$ that shift the energy distribution towards the EUV. For comparison, the visual absolute magnitudes of $\mathrm{O}$ type stars lie between $M_{\mathrm{v}} \approx$ -4.5 and $-7.5 \mathrm{mag}$ (Vacca et al. 1996).

It was clear from the first reports (Pollock 1987b) that WC stars are generally fainter in X-rays than other hot stars, the majority of measurements yielding merely upper limits. We have now taken advantage of the unprecedented sensitivity of XMM-Newton to observe the carbon type WC5 star, WR 114 (=HD 169010) for an exposure time, after excluding bad time intervals, of $15.9 \mathrm{ksec}$. To our knowledge, this is the first such sensitive exposure of a single WC star. Nevertheless, XMM-Newton was unable to obtain even a $2 \sigma$ detection of WR 114 in any energy band. In Sect. 2 we summarize the stellar parameters of WR 114 and in Sect. 3 we describe the observations of WR 114 with XMM-Newton. In Sect. 4 we review all currently available X-ray observations of single WC stars and in Sect. 5 we briefly review X-rays observations from binary WC stars. The results are discussed in Sect. 6 and conclusions are drawn in Sect. 7.

\section{Stellar parameters of WR 114}

WR 114 (WC5) is an ordinary representative of its spectral type. Its optical spectrum, the defining characteristic of the WC type, is well reproduced by the Potsdam standard atmospheric code, the latest version of which (Gräfener et al. 2002) we employed to infer the stellar parameters. The best fit to the observed spectrum, shown in Fig. 1, was obtained with an effective temperature $T_{*}=80 \mathrm{kK}$, luminosity $\log L_{\mathrm{bol}} / L_{\odot}=5.2$, mass loss rate $\log \dot{M}=-5.0 M_{\odot} \mathrm{yr}^{-1}$, terminal wind velocity $v_{\infty}=2000 \mathrm{~km} \mathrm{~s}^{-1}$, chemical composition He:C:O = 55:40:5 by mass, and a clumping contrast of $D=10$ (for definition of the latter see Hamann \& Koesterke 1998).

In "The vith Catalogue of Galactic WR Stars" (vanderHucht 2001) a number of galactic WC stars, including WR 114, are considered to have an OB companion because of the weakness of their emission lines in comparison with other stars of the same subtype. However, this "diluted emission line" or d.e.l. evidence is not very strong, as WR spectra depend on two parameters - effective temperature and mass-loss rate - and it is not clear a priori whether there exists a variety of line strengths within one spectral subclass. In Fig. 2 we compare the spectrum of WR 114 with the prototype apparently single WC5 WR 111, which was also one of the stars employed by van der Hucht (2001) for his comparison. The plot reveals that most spectral lines are very similar or even identical in strength and profile. Dilution by a companion's continuum would affect all lines uniformly. Therefore we doubt the "diluted emission line" case argued given by van der Hucht (2001) for the binary status of WR 114.

WR 114 was observed but detected neither in the midinfrared (Smith \& Houck 2001) nor as a source of non-thermal radio emission (Chapman et al. 1999), either of which would have provided supporting evidence for binarity. 


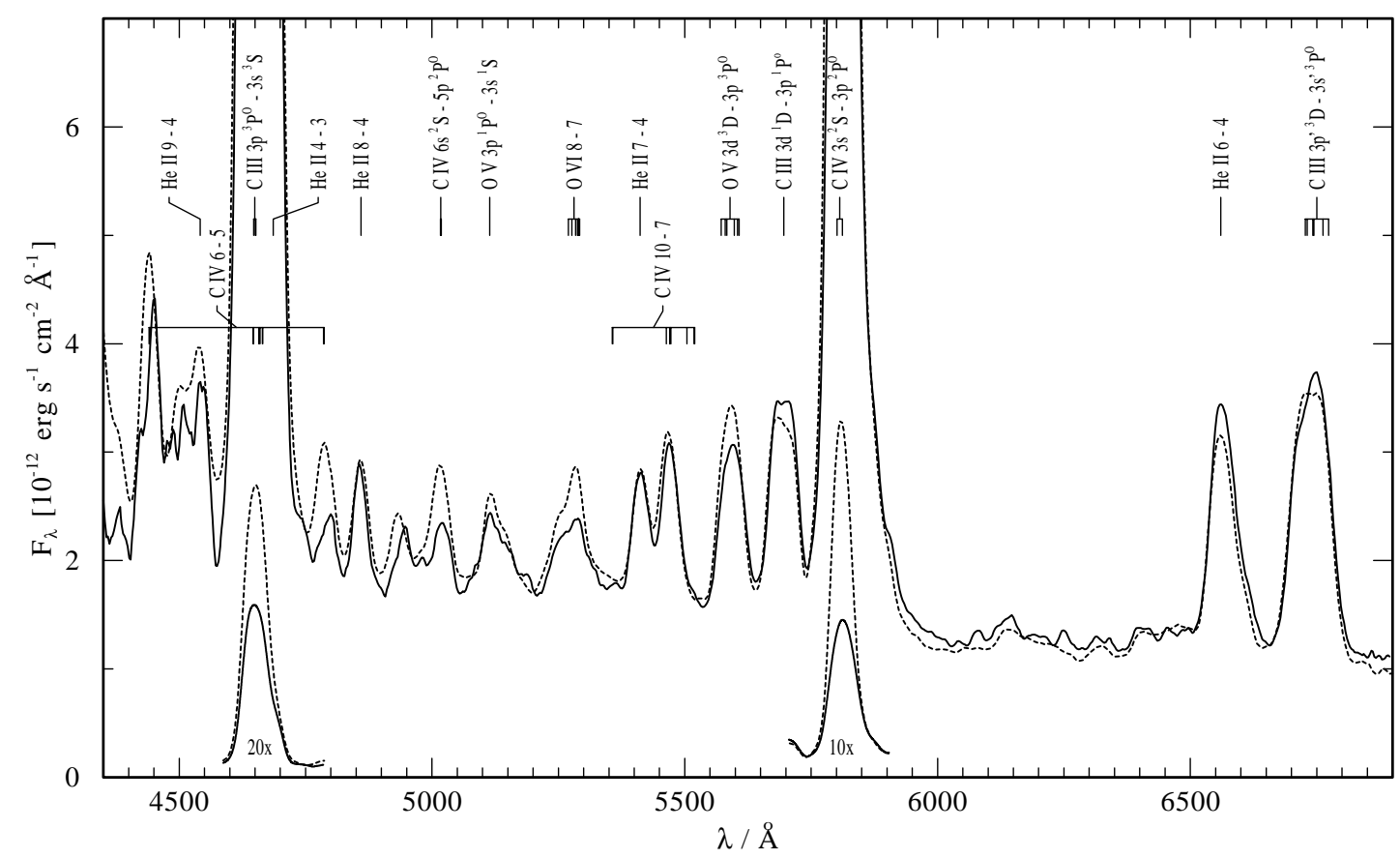

Fig. 2. Observed visual spectrum of WR 114 (solid line) from the atlas of Torres \& Massey (1987), compared to an observation of the single "standard" WC5 star WR 111 (dashed line). For this comparison, the WR 114 spectrum has been dereddened with $E_{b-v}=1.0$ mag in order to compensate for the stronger color excess of that star, and scaled by a factor of 3.0 to the flux level of WR 111 . Obviously, the spectra of both stars are very similar and indicate similar stellar parameters. Therefore we see no reason to suspect that the WR 114 spectrum is a binary composite, in contrast to the "diluted emission line" entry given in the van der Hucht (2001) catalogue.

\section{XMM-Newton observations of WR114}

XMM-Newton observed WR 114 on 23 March 2002, as summarized in Table 1 , for about $23.4 \mathrm{ksec}$ in total. Two exposures were taken of $16.9 \mathrm{ksec}$ and $6.5 \mathrm{ksec}$ although the latter was affected by a spacecraft pointing error and is unusable.

The observatory is described in a special letters issue of Astronomy \& Astrophysics (vol. 365, L1). Relevant data were obtained with the European Photon Imaging Camera (EPIC) and the Optical/UV Monitor (OM). EPIC is equipped with two identical MOS cameras and a pn camera. These provide CCD imaging spectroscopy with energy coverage between $0.2-15 \mathrm{keV}$ in a 30 arcmin diameter field-of-view (FoV) with an angular resolution of approximately $12 \operatorname{arcsec} F W H M$ at $1.5 \mathrm{keV}$. Observations were made using the full-window mode and the medium optical blocking filter. OM provides photometric coverage between $1700 \AA$ and $6500 \AA$ of the central 17 arcmin region of the $\mathrm{X}$-ray FoV, permitting simultaneous observations in both the X-ray and the UV/optical bands.

Preliminary examination of the standard pipeline processed images of the full $16.9 \mathrm{ksec}$ exposure immediately gave a puzzling result. Even though the star is obvious in the OM optical $V$ band image at an offset of only 0.02 from its expected position, as shown in Fig. 3, it is nowhere to be seen in any of the X-ray images such as the one shown in Fig. 4. The coordinates of the star and instrument are shown in Table 1 with other relevant data. We then performed a thorough data reduction using the XMM-Newton Science Analysis System software (SAS v 5.3.3). The latest calibration files were used and data were filtered to select good event patterns ensuring that only X-ray related events were considered. After exclusion of contaminated time intervals due chiefly to soft proton flares, the useful exposure time dropped from $16.9 \mathrm{ksec}$ to $15.9 \mathrm{ksec}$ for each of the MOS cameras and $10.2 \mathrm{ksec}$ for pn. The extracted images were carefully investigated. Some X-ray sources are obvious (see Fig. 4), but the area in the vicinity of the optical image of WR 114 contains no X-ray source in either MOS1, MOS2 or pn, individually or in combination. Exposure times, corrected for vignetting, were then determined for this area. Since there are no nearby X-ray or bright UV sources which could contaminate the image, and as the number of counts per pixel in the area is the order of unity, Poisson statistics are applied.

We used the SAS's source detection task EDETECT_CHAIN with the recommended $4 \sigma$ detection likelihood threshold of 10 . Attempts to detect the source were made both in narrow band and broad band images without success. Neither was WR 114 detected with thresholds as low as $2 \sigma$. Thus the conclusion is unavoidable that WR 114 was not detected by our XMM-Newton pointed observation.

We are able to set an upper limit on the X-ray count rate of WR 114. The on-axis $1.5 \mathrm{keV}$ point spread functions (PSFs) of the X-ray telescopes have $F W H M$ values of about 4.4 arcsec for MOS1, 2 and <12 arcsec for pn. The EPIC source positions, even for faint sources close to the detection limit, have typical $90 \%$ confidence radii of about $2-5$ arcsec, limited by the statistical accuracy of the measurements (Watson et al. 2001). Finally, one pixel in the EPIC CCDs covers about 1.1 arcsec for the MOS cameras and 4.1 arcsec for the pn. We chose to take a $3 \times 3$ pixel box covering $15 \times 15$ arcsec centered on the OM position of WR 114. Using the SAS task ESENMAP, we estimated the 
Table 1. XMM-Newton Observations of WR $114^{a}$.

\begin{tabular}{ll}
\hline \hline Parameter & Value \\
\hline Start (UT) & $2002-03-2313: 46$ \\
End (UT) & $2002-03-2318: 44$ \\
Exposure [ksec] & $15.9(\mathrm{MOS} 1,2), 10.2(\mathrm{pn})$ \\
Pointing coordinates & $18^{\mathrm{h}} 23^{\mathrm{m}} 16^{\mathrm{s}} 39-13^{\circ} 43^{\prime} 25^{\prime \prime} 8$ \\
WR114 coordinates & $18^{\mathrm{h}} 23^{\mathrm{m}} 16^{\mathrm{s}} .30-13^{\circ} 43^{\prime} 26^{\prime \prime} 0$ \\
Count Rate $\left[10^{-4} \mathrm{cnt} \mathrm{s}^{-1}\right]$ & $<2.6(\mathrm{MOS} 1,2),<6.7(\mathrm{pn})$ \\
Flux [erg cm $\left.\mathrm{s} \mathrm{s}^{-1}\right]$ & $<5.3 \times 10^{-15}$ \\
Luminosity $\left[\mathrm{erg} \mathrm{s}^{-1}\right]$ & $<2.5 \times 10^{30}$ \\
\hline
\end{tabular}

${ }^{a}$ One $6.4 \mathrm{ksec}$ exposures was of no scientific use since the manoeuvre to the target failed. The count rates are $1 \sigma$ vignetting-corrected source upper limits. The flux and luminosity are for the MOS1 upper limit in the $0.2-10 \mathrm{keV}$ range and have been corrected for interstellar absorption of $N_{\mathrm{H}} \approx 5.5 \times 10^{21} \mathrm{~cm}^{-2}$.

$1 \sigma$ count rate upper limits reported in Table 1. Following the concept of XMM-Newton for which each of the three detectors (MOS1, MOS2 and pn) is located in the focal plane of its own $\mathrm{X}$-ray telescope and observations are made simultaneously, we have to accept as an upper limit on the source the lowest count rate of all three detectors, namely MOS1 (see Table 1).

Of crucial influence to the X-ray luminosity determination is the interstellar absorption. Because no UV observation of WR 114 is available, we used the visual color index from the Potsdam model fit described above and shown in Fig. 1. The slope of the spectral energy distribution is matched best for a reddening of $E_{B-V}=1.45 \mathrm{mag}$. For the correlation between color excess and hydrogen column density, we adopt the value given in Groenewegen \& Lamers $(1989), N_{\mathrm{H}}=(3.8 \pm 0.9) \times$ $10^{21} E_{B-V} \mathrm{~cm}^{-2}$. Then the interstellar column density of neutral hydrogen is $N_{\mathrm{H}} \approx 5.5 \times 10^{21} \mathrm{~cm}^{-2}$ towards WR 114 .

The integrated X-ray flux, $F_{\mathrm{X}}$, can be estimated by multiplying the observed count rate, $\mathrm{CR}$, by an energy conversion factor ECF, that depends on the shape of the X-ray spectrum $F_{\mathrm{X}}=\mathrm{ECF} \times \mathrm{CR}$. To infer an upper limit on X-ray flux for WR 114, when there is no spectrum, we may only suggest some plausible model and treat the result with care. For OB stars, the ECF depends mainly on the X-ray temperature of the emitting plasma and the interstellar absorption (Berghöfer et al. 1996). We assume a temperature of $k T_{\mathrm{X}}=1 \mathrm{keV}$. Then the count rate is converted into flux using ECFs provided by the XMM-Newton Survey Science Center and the value of neutral hydrogen column density from our visual spectral fit. Since the distance to the star, $d=2 \mathrm{kpc}$, is known owing to the star's membership in the stellar association Sgr OB1 (van der Hucht 2001), we estimate an upper limit to the X-ray luminosity $(0.2-10 \mathrm{keV})$ of $L_{\mathrm{X}} \lesssim 2.5 \times 10^{30} \mathrm{erg} \mathrm{s}^{-1}$, yielding $\left(L_{\mathrm{X}} / L_{\text {bol }}\right) \lesssim 4 \times 10^{-9}$. It is necessary to emphasize that this upper limit is in fact the maximum XMM-Newton sensitivity for a given exposure time and level of internal and cosmic background.

Other sources were detected during our abortive attempt on WR114 as clear from the MOS1 image shown in Fig. 4 and the pn image in Fig. 6. Some of them are listed in Table 2. The optical counterparts were identified using "The STScI Guide Star

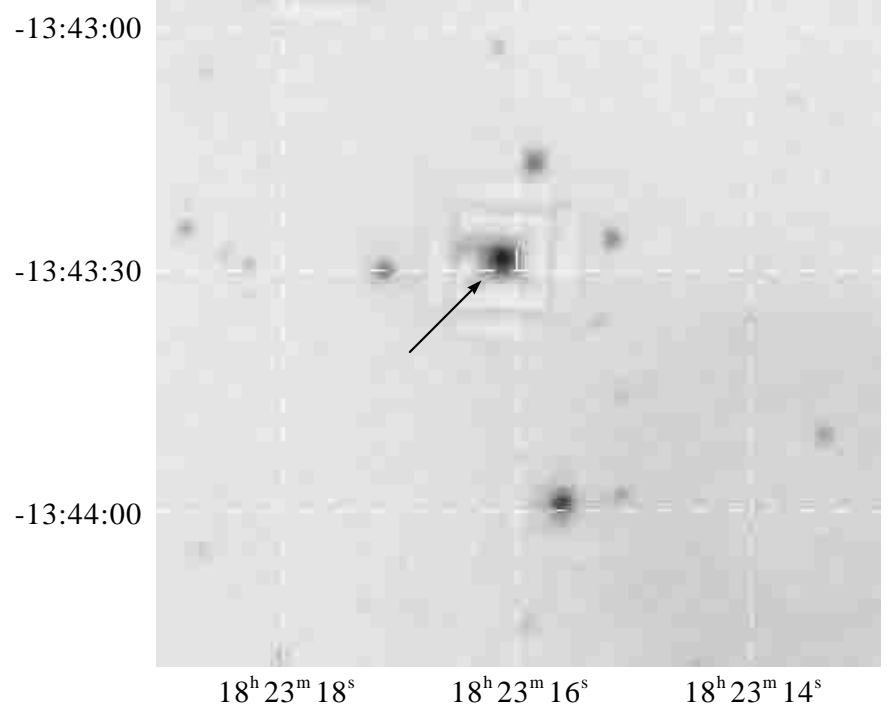

Fig. 3. Low-resolution OM image in $V$ band of WR 114. The star is clearly seen in the center of the image as indicated by the arrow.

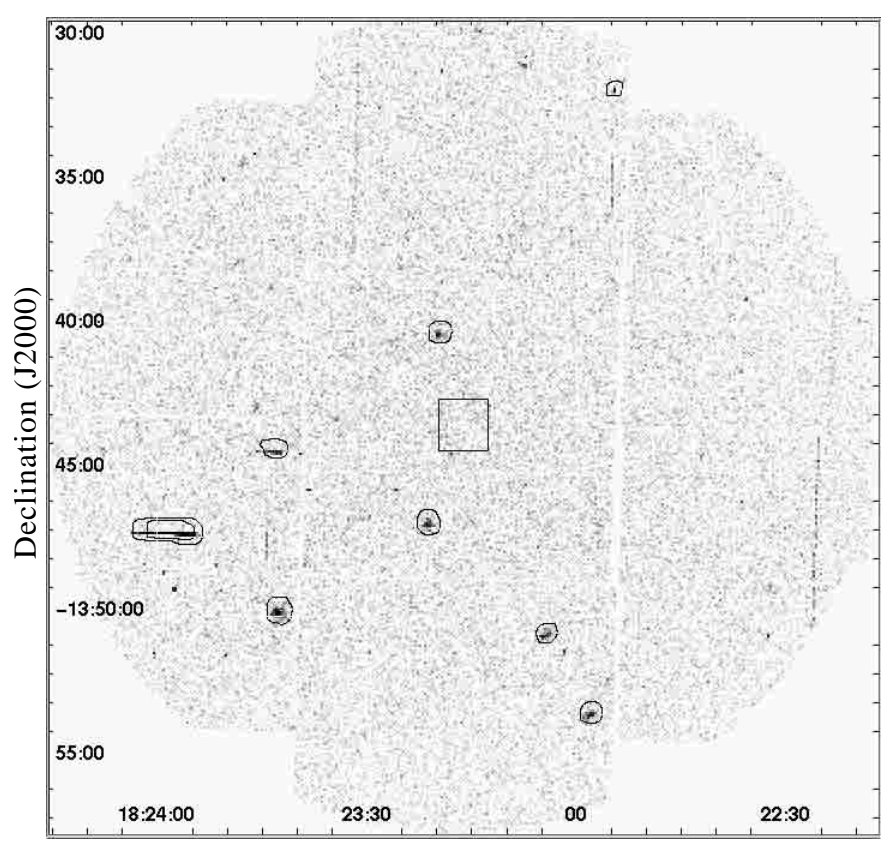

Right ascension (J2000)

Fig. 4. MOS1 X-ray image $(0.2-10 \mathrm{keV})$. The square box indicates the same area of sky as shown in Fig. 3.

Catalog 2.2" and the offsets between centers of X-ray images and optical images of the stars are 0.' 4 for XMMU J182342.8134949 and 0.' 1 for XMMU J182321.2-134645. The latter source is 1.4 arcmin from the pulsar PSR J1823-1347 which has no known optical counterpart although the much closer coincidence with a star of $V=14.33$ mag is probably more relevant. It is worth noting that X-ray sources with optical stellar counterparts have little emission above $2 \mathrm{keV}$ and are probably 
Table 2. Brightest X-ray sources detected by XMM-Newton in the vicinity of WR 114.

\begin{tabular}{ccc}
\hline \hline $\mathrm{XMMU}^{a}$ & $\begin{array}{c}\text { Count Rates } \\
{\left[\mathrm{cnt} \mathrm{s}^{-1}\right]}\end{array}$ & $\begin{array}{c}\text { Optical Counterpart } \\
{[\mathrm{mag}]}\end{array}$ \\
\hline $\mathrm{J} 182342.8-134949$ & $0.14 \pm 0.009$ & star, $V=13.36$ \\
$\mathrm{~J} 182321.2-134645$ & $0.05 \pm 0.005$ & star, $V=14.33$ \\
$\mathrm{~J} 182258.1-135324$ & $0.12 \pm 0.01$ & - \\
$\mathrm{J} 182304.3-135038$ & $0.06 \pm 0.005$ & - \\
$\mathrm{J} 182319.8-134011$ & $0.04 \pm 0.005$ & - \\
\hline
\end{tabular}

${ }^{a}$ According to the naming convention posted on the XMM-Newton website.

galactic while those without optical counterparts have no $\mathrm{X}$-rays below $2 \mathrm{keV}$ and are probably either young stellar objects or extragalactic.

\section{X-ray observations of single WC stars}

There has been steady progress in the study of stellar X-ray emission. The cornerstone imaging X-ray observatories of the 20th century - EInSTEIn (0.2-4.0 keV), Rosat (0.2-2.4 keV), and Asca (0.4-10 keV) - have now finished and the new generation observatories XMM-Newton $(0.2-15 \mathrm{keV})$ and CHANDRA $(0.08-10 \mathrm{keV})$ are in operation. With the completion of source catalogues from the EInstein, Rosat, and Asca missions, it is time to review the X-ray properties of WC stars.

In order to do so we searched all available catalogs of EinsteIn, Rosat, and Asca missions. The normal practice for the inclusion of a source in a catalogue is a likelihood detection threshold of 10 . Thus it is possible that real but weak detections might have been omitted. The Rosat All-Sky Survey Faint Source Catalogue (RASS-FSC) is derived from the allsky survey performed during the RosAT mission. The sources with a detection likelihood of at least 7 and containing at least 6 source photons are included in this catalogue.

In addition, we retrieved available archival data and performed our own data reduction with no detection threshold with the purpose of pinpointing even fainter sources. The results are discussed below.

Two sub-samples of WC stars from "The virth Catalogue of Galactic WR Stars" (van der Hucht 2001) were defined. The first group was formed by nominating as single stars those not designated as spectroscopic or colliding-wind binaries in the catalogue. We have thus counted the d.e.l. stars with the single stars for the assessment of WC star X-ray emission. There are 56 such stars in the vith catalogue. We note that amongst them, there are a few objects with the presence of dust as indicated by the letter " $d$ " in spectral classification. Also, one of the stars from our sample, WR 90 (WC7), is a confirmed source of nonthermal radio emission (Chapman et al. 1999). Therefore, it is most likely that other binary systems are to be found in this sub-sample as well as single stars. Nevertheless, none of these WC stars appears in any of the EInsteIn, Rosat or Asca source catalogues. It seems that the non-detection of WR 114 by XMM-Newton confirms the results of more than 20 years of attempts to observe X-rays from WC stars but to a greater degree of sensitivity.

The second sub-sample from the vith catalogue includes spectroscopic and colliding wind binaries. There are 31 such systems in the catalog. All X-ray sources included in EInstern, Rosat or Asca source catalogues amongst WC stars belong to this group.

We will now briefly discuss the archival observations of WC-type stars. The first quantitative information on the X-ray emission of hot massive stars was obtained by the EINSTEIN observatory. Seven single WC stars were observed with the IPC aboard EINSTEIN and none detected as shown in Table 3 taken from Pollock (1987b). It is usual practice to apply a source detection threshold of $\lambda>10$, far above any of the WC star statistics, which are quite typical of random background fluctuations. The exposure times achieved by EINSTEIN are quite significant, giving flux upper limits around $F_{\mathrm{X}} \lesssim 10^{-14} \mathrm{erg} \mathrm{cm}^{-2} \mathrm{~s}^{-1}$.

The results were further confirmed by the Rosat mission. The instruments, PSPC and HRI, aboard RosAT were very occasionally used to observe WC stars during both the pointed programme and the All-Sky Survey (RASS). Although pointed observations had relatively long exposure times, the survey had the merit of observing essentially all Galactic WR stars for a few hundred seconds.

As pointed out by Pollock et al. (1995) in a compilation of RosAT observations, single WR stars are weak X-ray sources in general, with only upper limits for the majority. There is no single WC star which is included in any of the RASS catalogues.

Since all of the data are now public we have extracted and analyzed the WC stars reported in Table 4. The table includes the visually brightest single WC stars and those WC stars which have available pointed observations. Once again, there are no detections to report. The apparent view in the literature that all types of WR stars are sources of X-ray emission can only conceivably have been based on Pollock et al.'s (1995) report of a weak detection of WR 111 using results from an early version of the Rosat pipeline. With the more rigorous analysis reported here, we are unable to confirm even that feeble result. The flux upper limit is roughly $F_{\mathrm{X}} \lesssim 3 \times 10^{-14} \mathrm{erg} \mathrm{cm}^{-2} \mathrm{~s}^{-1}$.

These long-standing doubts over the emission from single WC stars can now be confronted with the advent of more sensitive observations from the newer generation of instruments. Accordingly, we have retrieved all available Asca, XMM-Newton and Chandra archival data. For all the stars discussed, if not pointed out otherwise, the interstellar neutral hydrogen column density was obtained in the same way as for WR 114 (see above) using van der Hucht's (2001) values of $E_{b-v}$.

WR 111 (WC5). Asca images of weak diffuse X-ray emission near the area of sky where WR 111 is located are in the public domain. On 10 Oct. 1993 a $29 \mathrm{ksec}$ GIS exposure and a $23 \mathrm{ksec}$ SIS exposure were taken, with no detection of a point source anywhere in the vicinity of WR 111, as can be seen from Fig. 5. Asca was capable of $5 \sigma$ point source detections at flux limits as low as $\sim 4 \times 10^{-14} \mathrm{erg} \mathrm{cm}^{-2} \mathrm{~s}^{-1}$ (Tanaka et al. 1994). Although this value can be accepted as an upper limit for non-detected sources, we decided to obtain more precise 
Table 3. EINSTEIN observations of WC-type stars showing the log-likelihood detection statistic, $\lambda$, and the maximum-likelihood count rate.

\begin{tabular}{rrrcc}
\hline \hline WR & type & $\begin{array}{r}\text { time } \\
{[\mathrm{ksec}]}\end{array}$ & $\lambda$ & $\begin{array}{c}\text { Count Rate } \\
{\left[10^{-2} \mathrm{cnt} \mathrm{s}^{-1}\right]}\end{array}$ \\
\hline 5 & WC6 & 6.1 & 0.0 & 0.0 \\
17 & WC5 & 5.5 & 0.0 & 0.0 \\
57 & WC7 & 6.5 & 0.0 & 0.0 \\
111 & WC5 & 4.3 & 1.0 & 0.2 \\
135 & WC8 & 10.8 & 0.3 & 0.1 \\
144 & WC4 & 18.3 & 0.0 & 0.0 \\
154 & WC6 & 4.3 & 0.3 & 0.1 \\
\hline
\end{tabular}

Table 4. Rosat observations of prominent WC stars ${ }^{a}$.

\begin{tabular}{rcccccc}
\hline \hline WR & type & $\begin{array}{c}V \\
{[\mathrm{mag}]}\end{array}$ & $\begin{array}{c}\text { time } \\
{[\mathrm{ksec}]}\end{array}$ & $\begin{array}{c}\text { Count Rate } \\
{\left[10^{-3} \mathrm{cnt} \mathrm{s}^{-1}\right]}\end{array}$ \\
\hline 23 & WC6 & 9.67 & 2.8 & 0.7 & $0.5 \pm 0.2$ & PSPC \\
52 & WC5 & 9.86 & 0.2 & 2.4 & $0.0 \pm 3.0$ & PSPC \\
90 & WC7 & 7.45 & 0.3 & 0.0 & $0.0 \pm 4.0$ & PSPC \\
106 & WC9 & 12.33 & 15.7 & 0.0 & $0.0 \pm 0.5$ & PSPC \\
111 & WC5 & 8.23 & 18.9 & 3.0 & $0.9 \pm 0.4$ & PSPC \\
135 & WC8 & 8.36 & 11.0 & 2.1 & $2.1 \pm 1.7$ & PSPC \\
144 & WC4 & 15.49 & 81.3 & 0.0 & $0.0 \pm 0.1$ & HRI \\
154 & WC6 & 11.54 & 0.4 & 0.3 & $4.8 \pm 6.7$ & PSPC \\
\hline
\end{tabular}

${ }^{a}$ Rosat observations of prominent WC stars from either the short exposures of the PSPC all-sky survey or longer pointed observations with either the PSPC or the HRI detectors. The observation time reported is that combined from the one or more available pointings, often at different off-axis angles. The count rate is the mean.

estimates making use of standard processed files retrieved from the archive.

We obtained $1 \sigma$ upper limits on the count rate using the number of counts in the area which corresponds to the PSF, and the exposure time corrected for vignetting as listed in Table 5. As described in the Asca data reduction guide, for GIS at energies of about $1 \mathrm{keV}$, the $F W H M$ point spread function is about 3 arcmin. For SIS the PSF is smaller, about 1.5 arcmin. We used $1 \sigma$ limits for Gaussian statistics as tabulated in Gehrels (1986). Using stellar parameters for WR 111 from Gräfener et al. (2002), the upper limit to the X-ray flux, corrected for the interstellar absorption, is $F_{\mathrm{X}} \lesssim 5.0 \times$ $10^{-14} \mathrm{erg} \mathrm{cm}^{-2} \mathrm{~s}^{-1}$. Therefore, the upper limit on the X-ray luminosity of WR 111 is $\log L_{\mathrm{X}} / L_{\mathrm{bol}} \lesssim-7.8$.

WR 121 (WC9d). The vicinity of WR 121 was observed with Asca on 23 Mar 1999 for a $53 \mathrm{ksec}$ exposure time with GIS and $50 \mathrm{ksec}$ with SIS. There is an extended source of diffuse X-ray emission in the central part of the image (Fig. 5). The outer region of this diffuse source is superposed with the celestial coordinates of WR 121. Nevertheless, the signal from the diffuse source is very weak, and so we determine an upper limit of $F_{\mathrm{X}} \lesssim 2 \times 10^{-13} \mathrm{erg} \mathrm{cm}^{-2} \mathrm{~s}^{-1}$ for the unabsorbed flux. The stellar parameters of WR 121 are not well known. Therefore we adopted a typical luminosity of WC9 type stars of $\log L_{\mathrm{bol}} / L_{\odot}=5.3$ resulting in $\log L_{\mathrm{X}} / L_{\mathrm{bol}} \lesssim-7.4$.
WR 144 (WC4). This star was in the FoV of Asca observations of the Cygnus OB2 stellar association. During a $36.3 \mathrm{ksec}$ GIS exposure and $33 \mathrm{ksec}$ SIS exposure on 29 Apr. 1993 (Kitamoto \& Mukai 1996), the four point-like sources are the trapezium of O stars from Harnden et al.'s (1979) discovery paper. Within the $1 \sigma$ limit, there is no other point source in the image (Fig. 5). There is however a large amount of diffuse X-ray emission in Cyg OB2 which spreads out to the region where WR 144 is located. Because WR 144 is a highly reddened star, its stellar parameters are not wellknown. Using the stellar parameters of WR 111 and adopting $E_{B-V}=1.82 \mathrm{mag}$ from Massey et al. (2001), we estimate $F_{\mathrm{X}} \lesssim 1.5 \times 10^{-13} \mathrm{erg} \mathrm{cm}^{-2} \mathrm{~s}^{-1}$, yielding $\log L_{\mathrm{X}} / L_{\text {bol }} \lesssim-7.4$.

WR 60(WC8). WR 60 was in the field of view of the EPIC pn instrument on board XMM-Newton during observations of the Galactic Plane on 6-7 Feb. 2002. A total exposure of $\sim 12.7 \mathrm{ksec}$ was achieved. But since WR 60 is located at $\sim 7.2$ arcmin off-axis, the useful exposure time is only $2.49 \mathrm{ksec}$ as listed in Table 6 . There is no point-like source in this area in any of the energy bands, as seen in Fig. 6. Due to the large offset from the center of the image, the sensitivity is much smaller than in the case of WR 114 . We estimate a $1 \sigma$ upper limit to the count rate of $1.5 \times 10^{-3} \mathrm{cnt} \mathrm{s}^{-1}$. Then the unabsorbed flux is $F_{\mathrm{X}} \lesssim 1.2 \times 10^{-14} \mathrm{erg} \mathrm{cm}^{-2} \mathrm{~s}^{-1}$. The nominal luminosity of WC8 stars is $\log L_{\mathrm{bol}} / L_{\odot}=5.3$, giving $\log L_{\mathrm{X}} / L_{\mathrm{bol}} \lesssim-8.2$ for WR 60.

WR 118(WC9d). The star was in the FoV of XMM-Newton on 15 March 2002, with a useful exposure time of $3.8 \mathrm{ksec}$ for MOS and $3.9 \mathrm{ksec}$ for pn. There is no point-like source detected in the X-ray image in the area where WR 118 is located, as seen in Fig. 6. The $1 \sigma$ upper limit to the count rate in the pn detector is $5 \times 10^{-4} \mathrm{cnt} \mathrm{s}^{-1}$. From this we infer an unabsorbed flux of $F_{\mathrm{X}} \lesssim 4 \times 10^{-14} \mathrm{erg} \mathrm{cm}^{-2} \mathrm{~s}^{-1}$, and adopting a typical luminosity for WC9 type stars, we obtain $\log L_{\mathrm{X}} / L_{\text {bol }} \lesssim-7.4$.

WR 101a-o, WR 102a-l. There are six late-type WC stars in the Sgr A region of the Galactic Center, which are highly reddened $\left(E_{B-V} \approx 9.5 \mathrm{mag}\right)$. Little can be said about these poorly studied stars. The properties and evolution of massive stars in this region may be different (see e.g. Coker et al. 2002) due to the exceptional conditions in the vicinity of the central Black Hole. The region has been extensively observed by nearly all X-ray satellites including two CHANDRA exposures. We retrieved the archival CHANDRA data and performed a crude analysis. None of the X-ray point sources coincide with optical images of any of the WC stars in this region but the level of $\mathrm{X}$-ray background is very high. Thus, no additional information in our quest on intrinsic X-ray emission from single WC stars could be drawn from these extremely interesting objects.

The same holds for the members of the Quintuplet cluster, one of the three massive clusters projected within $50 \mathrm{pc}$ of the Galactic center (Figer et al. 1999), which contains at least $8 \mathrm{WC}$ stars. The stars are highly reddened $\left(E_{B-V} \approx 9.5 \mathrm{mag}\right)$ and many of them are likely surrounded by dusty envelopes. It is therefore not surprising that no X-ray emission associated with the intrinsic properties of the stellar winds has been reported. 

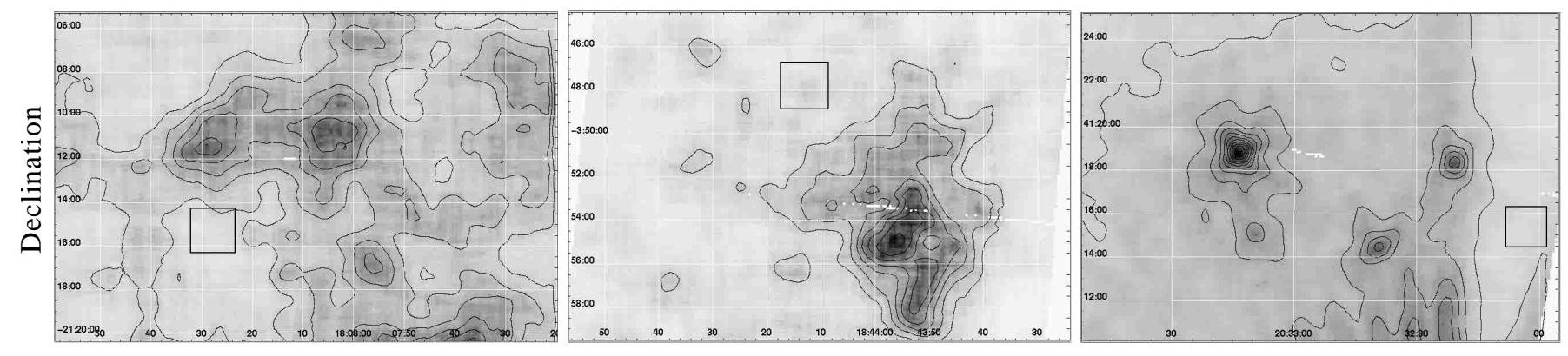

Right ascension

Fig. 5. ASCA SIS images of the areas of sky in the vicinity of WR 111, WR 121 and WR 144 (left to right). Areas which coincide with the celestial coordinates of the WR stars are denoted by black boxes. For the exposure times see Table 5.

Table 5. Asca observations of WC-type $\operatorname{stars}^{a}$.

\begin{tabular}{cccclc}
\hline \hline WR & $\begin{array}{c}\text { time } \\
{[\mathrm{ksec}]}\end{array}$ & \multicolumn{2}{c}{$\begin{array}{c}\text { Count Rates } \\
{\left[10^{-3} \mathrm{cnt} \mathrm{s}^{-1}\right]}\end{array}$} & $L_{\mathrm{X}} / L_{*}$ \\
\hline & $\mathrm{G}$ & $\mathrm{S}$ & $\mathrm{G}$ & $\mathrm{S}$ & \\
111 & 29 & 23 & 2.3 & 2.3 & $2 \times 10^{-8}$ \\
121 & 53 & 50 & 1.7 & 0.9 & $4 \times 10^{-8}$ \\
144 & 36 & 33 & 6.2 & 1.8 & $4 \times 10^{-8}$ \\
\hline
\end{tabular}

${ }^{a}$ Asca observations of WC-type stars showing $1 \sigma$ upper limits to S, the average of SIS1 and SIS2, and G, the average of GIS1 and GIS2, and the corresponding luminosity.

\section{X-ray observations of binary systems containing a WC star}

Extensive observational studies of WR stars in the Galaxy (see references in the vith Catalogue) suggest that the fraction of binaries among WC stars is quite large, probably more than half. Thus, it is likely that many WC-type stars are not yet detected binary systems of (a) two massive stars, usually WC+OB type, in which X-rays are generated by colliding stellar winds (e.g. Usov 1992), or rarely, (b) a WC star and a compact companion, such as Cyg X-3, with much more luminous accretion-powered X-rays.

Such is probably the case of WR 65 (WC9d). It is considered a binary in van der Hucht (2001) on the basis of the presence of diluted emission lines. We found that WR 65 is over-luminous for its spectral type: from our analysis of optical and UV spectra, we estimate $L_{\mathrm{bol}}=10^{6} L_{\odot}$. It is also a radio source, with indications of being a non-thermal radio emitter (Chapman et al. 1999).

Since WR 65 is 4 arcmin from one of the brightest X-ray pulsars in the Galaxy, PSR B1509-58, it has been in the FoV of nearly all X-ray satellites. However, until the excellent CHANDRA angular resolution became available, the star had not been resolved in X-rays. Chandra observed the pulsar wind nebula on 14 August 2000 for a $20 \mathrm{ksec}$ single exposure as reported in Gaensler et al. (2002). Figure 7 shows part of the CHANDRA's ACIS-I image of the region in the vicinity of PSR B1509-58, showing the point-like source X-ray source coinciding with WR 65. The neutral hydrogen column density derived by Gaensler et al. (2002) from fitting the X-ray spectrum of this point-like source, $N_{\mathrm{H}} \sim 3 \times 10^{22} \mathrm{~cm}^{-2}$, is quite
Table 6. XMM-Newton observations of WC-type stars reporting $1 \sigma$ count-rate and luminosity upper limits.

\begin{tabular}{|c|c|c|c|}
\hline WR & $\begin{array}{l}\text { time } \\
{[\mathrm{ksec}]}\end{array}$ & $\begin{array}{l}\text { Count Rates } \\
{\left[10^{-3} \mathrm{cnt} \mathrm{s}^{-1}\right]}\end{array}$ & $L_{\mathrm{X}} / L_{*}$ \\
\hline & MOS pn & MOS pn & \\
\hline 60 & 2.5 & $-\quad 3.3$ & $6 \times 10^{-9}$ \\
\hline 114 & $15.9 \quad 10.2$ & 0.60 .9 & $7 \times 10^{-10}$ \\
\hline 118 & $\begin{array}{ll}3.8 & 3.9\end{array}$ & 1.20 .5 & $4 \times 10^{-8}$ \\
\hline
\end{tabular}

close to the value we derived from the reddening of the visual and UV spectra of WR 65, with $E_{B-V}=2.42$ leading to $N_{\mathrm{H}} \approx 10^{22} \mathrm{~cm}^{-2}$.

The Chandra ACIS-I $5 \sigma$ detection yields a count rate of $(8.87 \pm 0.8) \times 10^{-3} \mathrm{cnt} \mathrm{s}^{-1}$, an unabsorbed flux of $F_{\mathrm{X}} \gtrsim 2 \times$ $10^{-13} \mathrm{~cm}^{-2} \mathrm{~s}^{-1}$, and $\log L_{\mathrm{X}} / L_{\mathrm{bol}} \gtrsim-6.8$. However, there is some ambiguity in the identification of X-ray point source in the ACIS-I image as WR 65. The X-ray source has an offset $\Delta \alpha=-0 . ' 009$ and $\Delta \delta=+1{ }^{\prime \prime} 63$ from WR 65 in FK5 (2000) coordinates. At the same time the radio coordinates of the source identified as WR 65 in (Chapman et al. 1999) differ from the $\mathrm{X}$-ray source in ChandRA's FoV by only $\Delta \alpha=0.003$ and $\Delta \delta=-0$.' 13 . It seems likely, that the radio and X-ray sources are identical. Gaensler et al. (2002) reported an uncertainty of \pm 0 .' 5 in each coordinate for the pulsar's X-ray position.

From broadband X-ray photometry it is already clear that WR 65 is a rather hard source, with an emission maximum in the range $2-4 \mathrm{keV}$ and only trace emission in softer passbands (see Fig. 6 from Gaensler et al. 2002). This is typical for the colliding wind binaries, such as $\gamma$ Velorum and WR 140, but hard to explain in terms of a single star. Skinner et al. (2002a) has reported on a hard component $(k T \geq 3 \mathrm{keV})$ in the spectrum of WR 110, a nitrogen-rich WN star that is believed to be single. However, in this case the dominant contribution to the $\mathrm{X}$-ray emission is rather soft with a characteristic temperature $k T \approx 0.5 \mathrm{keV}$. Therefore, combining data on the shape of $\mathrm{X}$-ray spectrum with radio and bolometric luminosity excess and the presence of dust we conclude that WR 65 is a newly discovered X-ray bright colliding wind system. It is clear that the nature of the X-ray emission of WR 65 should be verified by further observations. 


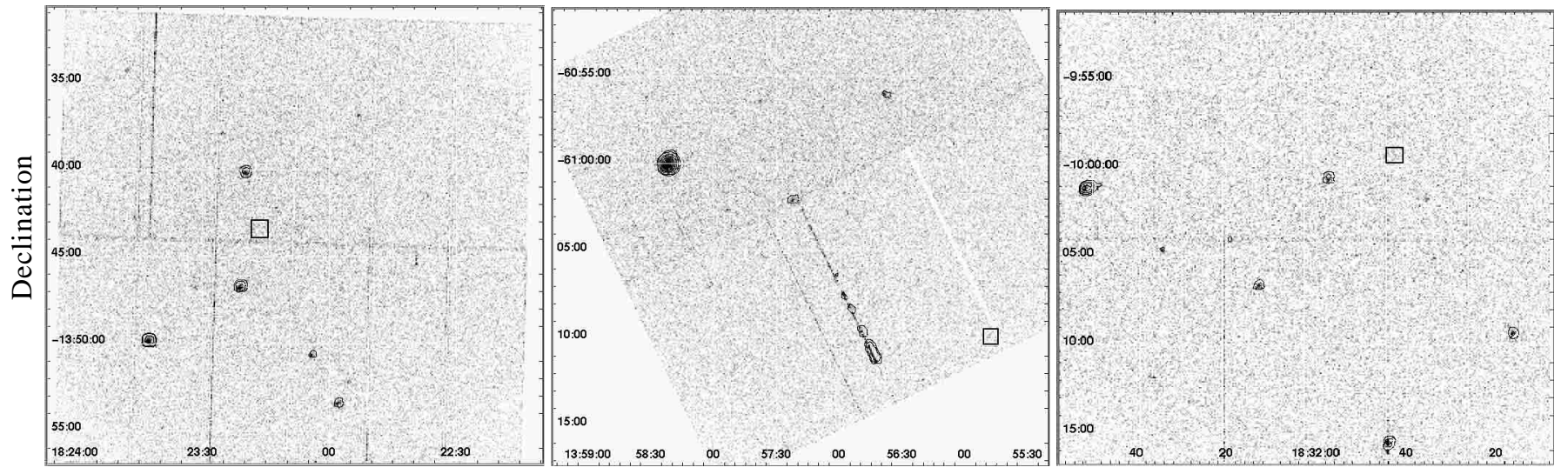

Right ascension

Fig. 6. XMM-Newton EPIC pn images near WR 114, WR 60 and WR 118, left to right, whose positions are at the centers of the black boxes. Exposure times are given in Table 6.

Although the mechanisms of X-ray production in binary systems are thought to be reasonably well understood, only 7 WC binary stars have been bright enough to be detected by one or more of the previous generations of X-ray instruments, namely WR 11, WR 48, WR 79, WR 93, WR 125, WR 137 and WR 140. All are spectroscopic binaries with orbital periods ranging between days and years. It looks as though we may now add WR 65 to their number. Seven WC stars located in the Sgr A and Quintuplet clusters have either ambiguous or false detections or large positional errors as indicated in object catalogues. Thus an overall fraction of detected Xray sources is $\approx 10 \%$ among all WC stars. Even among the total number of the 37 binary WC stars listed in the virth Catalogue, six deemed so only by virtue of diluted emission lines, about $20 \%$ are detected X-ray sources. In this regard, the weakness of the X-ray emission from WR 114 has little or no bearing on its possible binary status.

By far the brightest two X-ray WR binary systems, WR 140 (O4-5v+WC7) and $\gamma$ Velorum (WR 11, O7.5III+WC8), both have WC stars and have been observed reasonably often (e.g. Williams et al. 1990; Stevens et al. 1996; Zhekov \& Skinner 2000; Skinner et al. 2001). Both show variable absorption dependence on binary phase. WR140 is optically thin for most of its 7.94-year orbit, but shows strong absorption of X-rays near periastron. By contrast, absorption is heavy during most of $\gamma$ Velorum's 80-day orbit, reaching a still substantial minimum when the O-star is in front. XMM-Newton observations of $\gamma$ Vel have been reported by Dumm et al. (2002). At maximum light, the hard X-ray emission zone is clearly visible, though still absorbed below about $2 \mathrm{keV}$. At minimum light and throughout the orbit, a constant soft X-ray component is present that is convincingly explained by Dumm et al. (2002) as emission from cool, distant WC material photoionized by the central collision zone. In both systems, the WC star acts only as a passive X-ray absorber of dimensions of a few AU.

Thus, we may conclude from the available spectral and temporal data on binary systems, that there is little or no evidence for WC-type stars being intrinsic X-ray sources, and that WC stars are effectively opaque to X-rays from the colliding wind zone. The small percentage of WC binary X-ray sources shows that the conditions under which such a system can emit $\mathrm{X}$-rays are rather special, requiring particular combinations of orbital dimensions and viewing geometry.

\section{Discussion}

New spectral windows, larger and better telescopes, and advances in technology have led and continue to lead to important new discoveries about well-known astrophysical sources, and even whole new classes of sources. Such has also been the case with X-rays from hot massive stars.

Although there is no doubt that OB stars emit X-rays, neither the properties of the ensemble or individual spectra are well explained. The rough scaling relationship between bolometric and X-ray luminosity of OB stars $L_{\mathrm{X}} \propto 10^{-7} L_{\text {bol }}$ established with early EINSTEIN observations by Pallavicini et al. (1981) has been interpreted as a consequence of the coupling of the wind momentum-luminosity relation of Kudritzki et al. (1999) with a special distribution of hot plasma in the wind (Owocki \& Cohen 1999). The X-ray emitting WN stars do not follow this scaling law (Wessolowski 1996) although Ignace \& Oskinova (1999) showed that the measurements could be understood if the X-ray plasma filling factor depends on mass loss rate and terminal velocity.

Whereas EINSTEIn, Rosat and Asca could provide only gross energy distributions, CHANDRA and XMM-Newton have been able to resolve individual lines and thus challenge further the current view of X-ray production in radiatively-driven winds. The theoretical X-ray spectra of hot stars with winddistributed shocks are expected to produce asymmetric emission line profiles with blueshifted emission peaks owing to wind absorption effects (e.g. Ignace 2001; Owocki \& Cohen 2001; Ignace \& Gayley 2002). Recent results from CHANDRA and XMM-Newton have shown that most of the OB stars observed so far display symmetric and unshifted emission profiles (e.g. Schulz et al. 2000; Waldron \& Cassinelli 2001). Although the $\mathrm{O}$ star $\zeta$ Pup is a notable exception, with profiles better matching theoretical expectations (Kahn et al. 2001; 


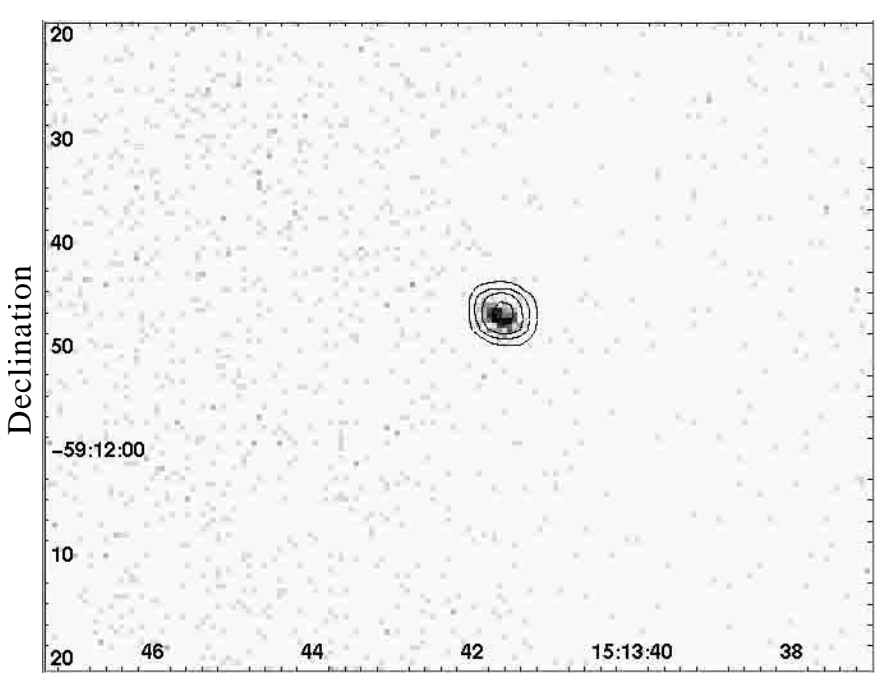

Right ascension

Fig. 7. Part of the Chandra ACIS-I image of the Hit region in the vicinity of the pulsar PSR B1509-58. The point-like X-ray source is 1".6 from the optical position of WR 65.

Cassinelli et al. 2001), it has not yet been possible to explain symmetric line profiles from single stars in the framework of the shock model.

The WR stars are another class of object in which the $\mathrm{X}$-ray emission is thought to arise from a similar mechanism. The WR stars, being fainter, are an integral step behind the OB stars in terms of spectral resolution. RosaT and Asca provided passband fluxes for WR stars, and now XMM-Newton is providing spectral shapes. The characteristic feature of embedded shock models is the soft X-ray spectrum, with maximum temperatures corresponding to a fraction of terminal velocity of the wind. The emission is not expected to be present at energies harder than $\sim 2-3 \mathrm{keV}$. However, Skinner et al. (2002a,b) have reported "hard tails" (i.e., the presence of emission with $k T_{\mathrm{X}} \geq 3 \mathrm{keV}$ ) in the spectra of the WN stars WR 6 and WR 110 . We have observed WR 1 , which is of similar subtype as these other two WN stars. WR 1 does not appear to have a hard tail; however, the exposure for this source was less than for WR 6 and WR 110 , so that the existence of a hard tail in WR 1 is inconclusive at this point (Ignace et al., in prep.). The hard tail may indicate the presence of non-degenerate companions which have otherwise gone undetected in these systems, an option that is also discussed in an attempt to explain the X-ray line profiles in $\mathrm{O}$ stars.

In contrast to $\mathrm{OB}$ and $\mathrm{WN}$ stars, this paper reports on the effective absence of X-ray emission from WC stars, another unexpected result. The obvious question is, why are these stars so faint in X-rays? There are two obvious alternatives:

(1) WC stars are intrinsically inefficient in producing $\mathrm{X}$-rays. If we understood the production of X-rays by radiatively-driven winds better, it would be easier to assess this possibility. Our understanding is hampered by the fact that time-dependent hydrodynamical simulations including multiline scattering - the latter being central both for the wind driving and instability growth rates - are not available yet.
(2) X-rays are generated in the stellar winds of WC stars (as it is the case in OB star winds) but the strongly enhanced density and metal content of their winds lead to their total absorption before they can emerge to the observer.

The high optical depth for X-rays in WR winds has long been recognized (Pollock 1987a), and it is this possibility that seems the most likely. As an $\mathrm{O}$ star evolves through the WN stage, it converts $4 \mathrm{H} \rightarrow \mathrm{He}$ with an enhancement of $\mathrm{N}$ as the wind density increases. Both factors independently cause the X-ray opacity to rise. This is especially pronounced in cases where helium recombines to HeII, primarily in the winds of late type WN stars. The HeIr edge for those stars is strong enough to place the $\tau=1$ radius to several hundred stellar radii for the whole soft X-ray band.

For the WC stars, the abundance of CNO elements is drastically increased (while abundances of heavier elements like $\mathrm{S}$, $\mathrm{Si}$ and $\mathrm{Fe}$ are unchanged) and the mass loss rates are the same order as for WN stars. It is clear that such a metal-rich and dense medium is very efficient in absorbing X-rays, making $\mathrm{WC}$ winds even more opaque to $\mathrm{X}$-rays than the winds of $\mathrm{O}$ and WN stars.

We have checked this by means of our advanced non-LTE atmosphere model, accounting for line blanketing and clumping in a first approximation. Although the latter effect leads to smaller empirical mass loss rates (Hamann \& Koesterke 1998) compared with those found previously from homogeneous models, the radius of optical depth unity is still extremely large. As seen in Fig. 8, although HeII remains the major absorbing element for the soft X-rays, the high abundances of CNO group elements leads to strong K-shell absorption edges and places the $\mathrm{X}$-ray photosphere at distances exceeding a thousand stellar radii for photons with energies around $1 \mathrm{keV}$.

The upper limit of X-ray luminosity for WR 114 derived in this paper refers to the emergent stellar radiation after correcting for interstellar absorption. We cannot infer anything about the possible generation of X-rays by any hot plasma embedded deep in the wind that would be absorbed before they can escape. There is much room for such internal X-ray absorption as the optical depths in the wind of WR 114 is high to large distances. Without any knowledge about the location of the possible X-ray production and the detailed structure of the absorbing cool wind, quantitative limits on the internal X-ray production rate would be rather speculative.

It would be extremely difficult for the theory of instabilities in radiatively driven winds to explain the presence of $\mathrm{X}$-ray emitting material at distances of a thousand stellar radii. There are few detailed physical studies of the X-ray emission produced at such large radii. Feldmeier et al. (1997a,b) performed hydrodynamical simulations of $\mathrm{O}$ type star winds for radii up to $100 R_{*}$. The hot $\mathrm{X}$-ray emitting gas can be generated by shocks only in the accelerating part of the stellar wind, which does not extend further than $5-10 R_{*}$. When the expanding material reaches the constant velocity regime there are still regions of hot gas confined between dense and cold clumps. Nevertheless, the density of the interclump medium is 2-3 orders of magnitude lower than the density of clumps, hence cooling is not efficient and no X-rays can be generated. 


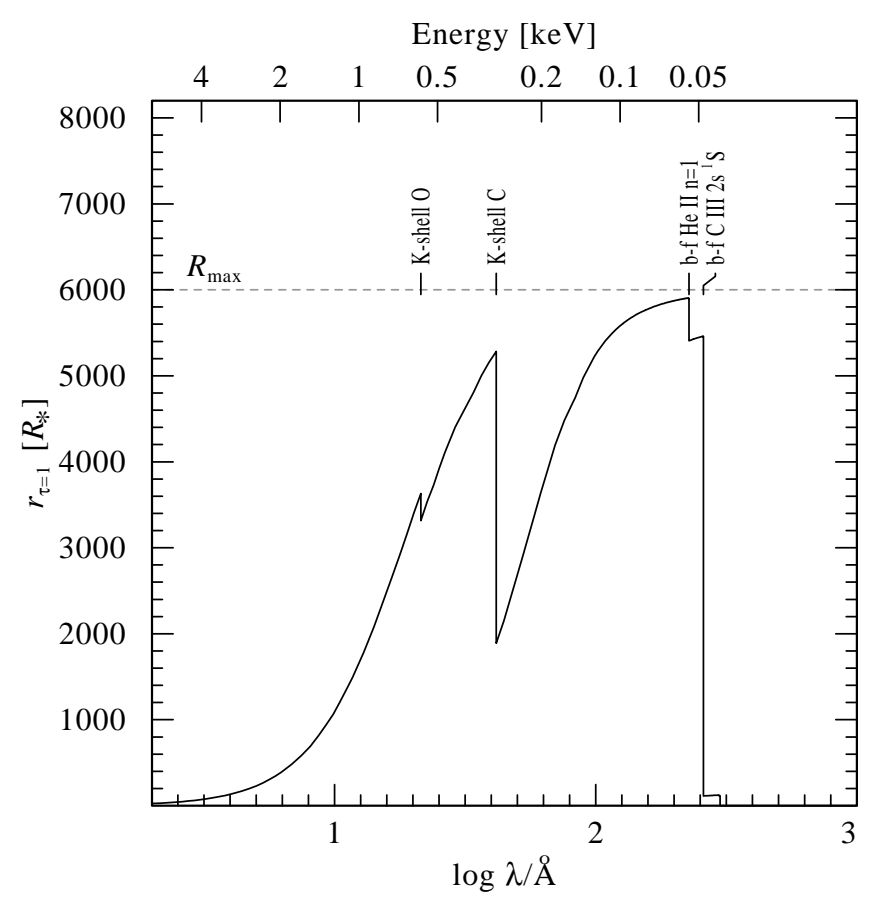

Fig. 8. Radius where the photoabsorbing optical depth is unity to X-rays for the WC star WR 114 as obtained from the Potsdam atmosphere code. The model parameters are as given in Sect. 2. The lower horizontal axis is for wavelength, and the upper is for energy. The most prominent He II edge at $228 \AA$ remains optically thick to the outer boundary of our model at $R_{\max }=6000 R_{*}$.

Runacres \& Owocki (2002) confirmed the major conclusions of Feldmeier et al. (1997a,b) and pointed out that the clumpy structure of the wind persists to outer regions with radii up to $100 R_{*}$ and is maintained by collisions between clumps moving with different speeds, at least in a 1D approach. Clumpclump collisions in the outer wind could hardly be a means for generating X-ray emitting plasma, since the relative velocities of the clumps are most likely not high enough. The slight chance that slowly approaching clumps can squeeze the thin hot gas between them to reach the densities relevant for X-ray emission should be explored via hydrodynamical simulations, but likely it cannot serve as the main mechanism for the hot plasma production.

There is no study known to us which would trace the evolution of wind structures as far out as the X-ray photosphere of WC stars. In any case, from the discussion above it seems implausible that X-rays could be generated so far out in the stellar wind. Thus, although from this point-of-view, the absence of $\mathrm{X}$-rays emerging from $\mathrm{WC}$ stars is consistent with the theory of radiatively driven winds, the wealth of new high-resolution spectroscopic data will undoubtedly stimulate future theoretical developments of the X-ray production in hot star winds.

\section{Conclusions}

We have analyzed the available X-ray archival data of WC stars and a $15.9 \mathrm{ksec}$ XMM-Newton observation of WR 114. The main points are:

(I) No single WC star has previously been detected as an $\mathrm{X}$-ray source, independent of spectral sub-type or other stellar parameters.
(II) XMM-Newton observation of the typical WR star WR 114 (WC5) confirmed that this carbon enriched Wolf-Rayet star does not emit X-rays above the detection limit of our observation. The upper limit to the X-ray flux from WR 114 is $F_{\mathrm{X}} \lesssim 5 \times 10^{-15} \mathrm{erg} \mathrm{cm}^{-2} \mathrm{~s}^{-1}$.

(III) The apparent absence of X-rays can be explained by the large absorptive opacity of the stellar wind from WC stars: the $\mathrm{X}$-ray photosphere is at a few thousand stellar radii.

(Iv) All WC stars that are currently known to emit X-rays are binary systems. The fraction of X-ray sources among all binary systems containing a WC star is less than $20 \%$, indicating that the conditions are special under which they are visible.

(v) A previously unidentified source of X-ray emission is attributed to the WC9d type star WR 65. Because of the hardness of its X-rays, we suggest that it too is a binary system.

Acknowledgements. We acknowledge useful discussions with A. Feldmeier and G. Gräfener. We wish to thank I. Antokhin for the guidance on using XMM-Newton's SAS. This research has made use of NASA's Astrophysics Data System Bibliographic Services and of the SIMBAD database, operated at CDS, Strasbourg, France. Data were obtained through the High Energy Astrophysics Science Archive Research Online Service, provided by the NASA/Goddard Space Flight Center. LMO acknowledges support for this research from the Deutsche Forschungsgemeinschaft grant Fe 573/1-1. RI acknowledges support for this research from NASA grant NAG5-12557. RI and JCB would like to thank PPARC for the support and LMO is grateful for the NATO/RS fellowship.

\section{References}

Berghöfer, T. W., Schmitt, J. H. M. M., \& Cassinelli, J. P. 1996, A\&AS, 118, 481

Castor, J. I., Abbott, D. C., \& Klein, R. I. 1975, ApJ, 195, 157

Cassinelli, J. P., Miller, N. A., Waldron, W. L., et al. 2001, ApJ, 554, L55

Chapman, J. M., Leitherer, C., Koribalski, B., et al. 1999, ApJ, 518, 890

Coker, R. F., Pittard, J. M., \& Kastner, J. H. 2002, A\&A, 383, 568

Corcoran, M. F., Swank, J. H., Petre, R., et al. 2001, ApJ, 562, 1031

Dumm, T., Güdel, M., Schmutz, W., et al. 2002, in Proc. Symp. New Visions of X-ray Universe in the XMM-Newton and Chandra Era, ed. F. Jansen, ESTEC, The Netherlands, in press

Feldmeier, A., Kudritzki, R. P., Palsa, R., et al. 1997a, A\&A, 320, 899 Feldmeier, A., Puls, J., \& Pauldrach, A. W. A. 1997b, A\&A, 322, 878 Figer, D. F., McLean, I. S., \& Morris, M. 1999, ApJ, 514, 202

Gaensler, B. M., Arons, J., Kaspi, V. M., et al. 2002, ApJ, 569, 878

Gayley, K. G., \& Owocki, S. P. 1995, ApJ, 442, 296

Gehrels, N. 1986, ApJ, 303, 336

Gräfener, G., Koesterke, L., \& Hamann, W.-R. 2002, A\&A, 387, 244

Groenewegen, M. A. T., \& Lamers, H. J. G. L. M. 1989, A\&AS, 79, 359

Hamann, W.-R., \& Koesterke, L. 1998, A\&A, 335, 1003

Harnden, F. R. Jr., Branduardi, G., Elvis, M., et al. 1979, ApJ, 234, L51

Hasinger, G., Altieri, B., Arnaud, M., et al. 2001, A\&A, 365, L45

van der Hucht, K. A. 2001, The vir th Catalogue of Galactic Wolf-Rayet Stars, New Astron. Rev., 45, 135

Ignace, R., \& Oskinova, L. M. 1999, A\&A, 348, L45

Ignace, R. 2001, ApJ, 549, L119

Ignace, R., \& Gayley, K. G. 2002, ApJ, 568, 954

Jansen, F., Lumb, D., Altieri, B., et al. 2001, A\&A, 365, L1 
Kahn, S. M., Leutenegger, M. A., Cottam, J., et al. 2001, A\&A, 365, L312

Kitamoto, S., \& Mukai, K. 1996, PASJ, 48, 813

Kudritzki, R. P., Puls, J., \& Lennon, D. J. 1999, A\&A, 350, 970

Lamb, D. H., Warwick, R. S., Page, M., et al. 2002, A\&A, 389, 93

Lucy, L. B., \& White, R. L. 1980, ApJ, 241, 300

Lucy, L. B. 1982, ApJ, 255, 286

Massey, P., Degioia-Eastwood, K., \& Waterhouse, E. 2002, AJ, 121, 1050

Owocki, S. P., \& Cohen, D. H. 1999, ApJ, 520, 833

Owocki, S. P., \& Cohen, D. H. 2001, ApJ, 559, 1108

Pallavicini, R., Golub, L., Rosner, R., et al. 1981, ApJ, 248, 279

Pollock, A. M. T. 1987a, A\&A, 171, 135

Pollock, A. M. T. 1987b, ApJ, 320, 283

Pollock, A. M. T., Haberl, F., \& Corcoran, M. F. 1995, Proc. IAU Symp. 163, ed. K. A. van der Hucht, \& P. M. Williams (Dordrecht: Kluwer), 191

Runacres, M. C., \& Owocki, S. P. 2002, A\&A, 381, 1015

Schulz, N. S., Canizares, C. R., Huenemoerder, D., \& Lee, J. 2000, ApJ, 545, L135

Seward, F. D., Forman, W. R., Giaconni, R., et al. 1979, ApJ, 234, L55
Skinner, S. L., Güdel, M., Schmutz, W., \& Stevens, I. R. 2001, ApJ, 558, L113

Skinner, S. L., Zhekov, S. A., Güdel, M., \& Schmutz, W. 2002a, ApJ, 572,477

Skinner, S. L., Zhekov, S. A., Güdel, M., \& Schmutz, W. 2002b, ApJ, 579,764

Smith, J. D. T., \& Houck, J. R. 2001, AJ, 121, 2115

Stevens, I. R., Corcoran, M. F., Willis, A. J., et al. 1996, MNRAS, 283, 589

Tanaka, Y., Inoue, H., \& Holt, S. S. 1994, PASJ, 46, L37

Torres, A. V., \& Massey, P. 1987, ApJS, 65, 59

Usov, V. V. 1992, ApJ, 389, 635

Vacca, W. D., Garmany, C. D., \& Shull, M. J. 1996, ApJ, 460, 914

Waldron, W. L., \& Cassinelli, J. P. 2001, ApJ, 548, L45

Watson, M. G., Auguères, J.-L., Ballet, J., et al. 2001, A\&A, 365, L51

Wessolowski, U. 1996, MPE Rep., 263, 75

Williams, P. M., van der Hucht, K. A., Pollock, A. M. T., et al. 1990, MNRAS, 243, 662

Woosley, S. E., Heger, A., \& Weaver, T. A. 2002, Rev. Mod. Phys., 74, 1015

Zhekov, S. A., \& Skinner, S. L. 2000, ApJ, 538, 808 